\title{
Technology Helps Students Transcend Part-Whole Concepts
}

Author(s): Anderson Norton, Jesse L. M. Wilkins, Michael A. Evans, Kirby Deater-Deckard, Osman Balci and Mido Chang Source: Mathematics Teaching in the Middle School, Vol. 19, No. 6, FOCUS ISSUE: Rational Number Sense (February 2014), pp. 352-358 Published by: National Council of Teachers of Mathematics Stable URL: http://www.jstor.org/stable/10.5951/mathteacmiddscho.19.6.0352 Accessed: 08-02-2017 19:08 UTC

JSTOR is a not-for-profit service that helps scholars, researchers, and students discover, use, and build upon a wide range of content in a trusted digital archive. We use information technology and tools to increase productivity and facilitate new forms of scholarship. For more information about JSTOR, please contact support@jstor.org.

Your use of the JSTOR archive indicates your acceptance of the Terms \& Conditions of Use, available at http://about.jstor.org/terms

National Council of Teachers of Mathematics is collaborating with JSTOR to digitize, preserve and extend access to Mathematics Teaching in the Middle School 

fractions. However, this foundation remains insufficient for mastering fraction concepts that students will see in the $\mathrm{K}$-grade 12 curriculum. In particular, part-whole analyses are meaningless when improper fractions, such as 7/5, are discussed. After all, how can a student make sense of 7 out of 5 ?

To work with the limitation, students might convert improper fractions to mixed numbers (e.g., 1 and 2/5), but they should also develop understandings that enable them to consider fractions as "numbers in their own right" (Hackenberg 2007, p. 27). Moreover, fraction operations, such as multiplication and division, can be cumbersome or even unintelligible unless students progress beyond part-whole concepts. For this reason, the Common Core State Standards for Mathematics (CCSSM) calls on teachers to support student development beyond partwhole conceptions, even as early as the third grade (CCSSI 2010). Studies of students' fractions knowledge indicate that such comprehensions are uncommon, even among middle school students (Hackenberg 2007; Norton and Wilkins 2010; Olive and Vomvoridi 2006; Steffe and Olive 2010).

\section{PARTITIONING AND ITERATING}

Partitioning and iterating are fundamental mental actions, or operations, that underlie fractions concepts. The limitation of part-whole concepts stems from a reliance on partitioning alone. Partitive thinking, on the other hand, relies on both partitioning and iterating. Of course, it is important that students are able to partition a continuous whole into a specified number of equal parts, as in sharing a candy bar among five friends. However, students should also understand that any one of those parts could be iterated, or multiplied, to produce other fractional parts. For

Fig. 1 These tasks, which assess partitive conceptions of unit fractions, require students to iterate a unit fractional part within the given whole.

What fraction is the smaller pie piece out of the whole pie?

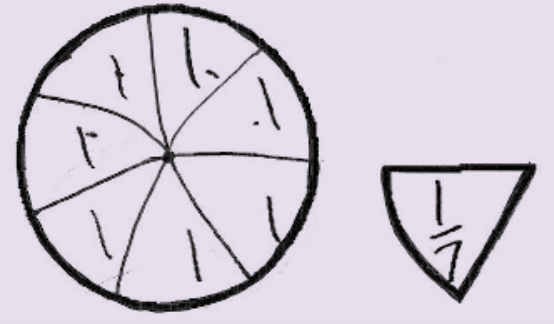

(a)

What fraction is the smaller stick out of the longer stick?

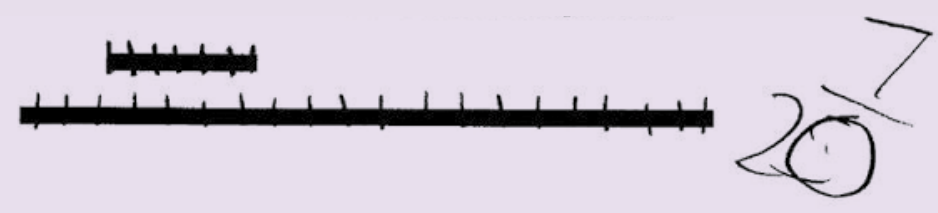

(b)

example, $3 / 5$ can be generated with three iterations of $1 / 5$. In a comparative analysis of international texts, Watanabe (2009) found that U.S. textbooks focus almost exclusively on part-whole tasks.

In contrast, tasks in Chinese and Japanese textbooks often refer to nonunit proper fractions as multiples of unit fractions; for example, $3 / 4$ is referred to as three times $1 / 4$. Although numerous differences are found in culture and education that contribute to stronger mathematics performance among students in China and Japan, it is important to consider textbooks as a contributing factor, especially because "in every country, mathematics textbooks exert a considerable influence on the teaching and learning of mathematics" (Howson 1995, pp. 5-6).

\section{ASSESSING STUDENTS' PARTITIVE CONCEPTIONS}

Students' development of partitive conceptions traces a learning trajectory outlined by Steffe and Olive (2010). Students begin by develop- ing partitive understandings for unit fractions, meaning that they can think about unit fractions as sizes relative to a specified whole. For example, students might begin to understand $1 / 5$ as a length that, when iterated (copied or multiplied) five times, reproduces the whole. Later on, students begin to generalize that knowledge and extend it to nonunit proper fractions so that, for instance, they can understand $3 / 5$ as a length that results from three iterations of $1 / 5$. Conceiving of improper fractions as multiples of unit fractions requires further development.

Figure 1 shows two sample tasks that we have used in assessing students' partitive knowledge of unit fractions. The responses are from two seventh-grade students. In a recent study in a small, rural school, we found that about 40 percent of seventh-grade students still struggle with such tasks (Norton and Wilkins 2010). Further, we have found that students who have constructed partitive conceptions of fractions are poised to construct more advanced 
Fig. 2 These research examples demonstrate students' constructions of partitive schemes.

Make a stick that is two times as big as this stick . . . three times . . . four times. . . .

(a)

If this stick is $1 / 7$ of a whole stick, can you show me how big the whole stick is?

(b)

ideas about fractions. These findings, which demonstrate that we cannot take partitive knowledge for granted, underscore the need for teachers to support this development.

Each task in figure 1 requires students to iterate a unit fractional part within the given whole to determine its unit-fractional size relative to that whole. The response associated with the task in figure 1a indicates that the student could name the unit fractional part based on the number of iterations within the whole. This is the essence of partitive reasoning, so we can infer that the student had at least begun to develop partitive conceptions for unit fractions.

The response associated with the task in figure $\mathbf{1 b}$ indicates that the student had not begun that same development with unit fractions. Instead, the response suggests a trial-and-error process of dividing the whole into parts that coincide with those on the smaller piece and then using a part-whole scheme to determine the fraction associated with the smaller piece-the number of parts out of the whole taken up by the smaller piece. In either case, the students' teachers should consider ways to further support that development.

\section{PROMOTING PARTITIVE UNDERSTANDING}

Fortunately, research tells us how we can support students' development of partitive understanding. From his research with a pair of fourth-grade students named Jason and Laura, Steffe (2001) found that he could support their developing knowledge base by engaging them in tasks involving the production of connected multiples from a given unit. For example, the task in figure $2 \mathrm{a}$ requires students to iterate, or make connected copies of, the given length to form new lengths.

Steffe concluded that such tasks create a bridge between students' use of iterating in discrete, whole-number contexts to their use of iteration in continuous, fractional contexts. Olive and Vomvoridi (2006) achieved similar results with a sixth-grade student named Tim by engaging him in tasks involving the production of a whole from a given unit fractional part. For example, the task in figure $\mathbf{2 b}$ requires students to iterate the given $1 / 7$ stick seven times to produce the whole from which it came.

In general, tasks intended to support conceptual learning should elicit students' available ways of operating (such as partitioning and iterating) and challenge them to coordinate those mental actions in new ways (Olive 2000). Furthermore, students should have opportunities to assess the success of those new ways of operating and to reflect on them (Simon and Tzur 2004). The educational game we present here was designed with those principles in mind.

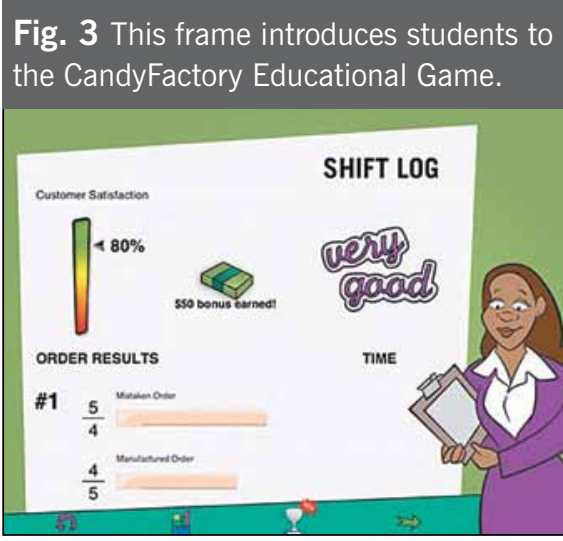

Fig. 4 CandyFactory's different fraction activities allow students to become familiar with different representations.

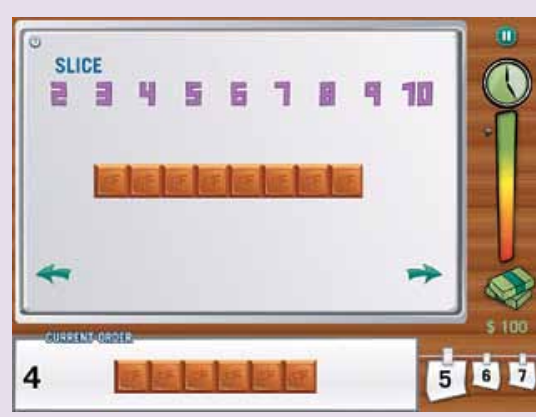

(a)

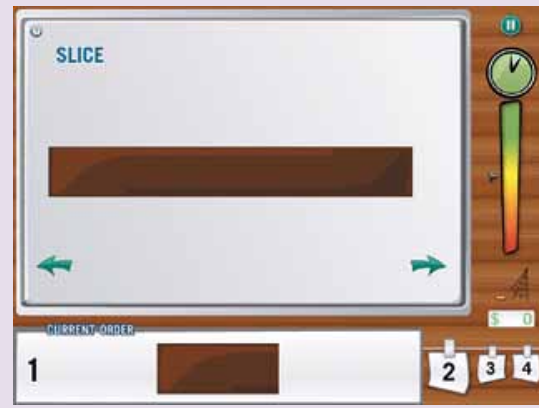

(b)

USING THE CANDYFACTORY APP With funding from the National Science Foundation, the Learning Transformation Research Group at Virginia Tech has designed an app to promote an understanding of partitive concepts through game play. This free app, CandyFactory Educational Game, is available for the $\mathrm{iPad}^{\circledR}$ on the iTunes ${ }^{\circledR}$ store. An older version of the app (under the same name) is also free and 


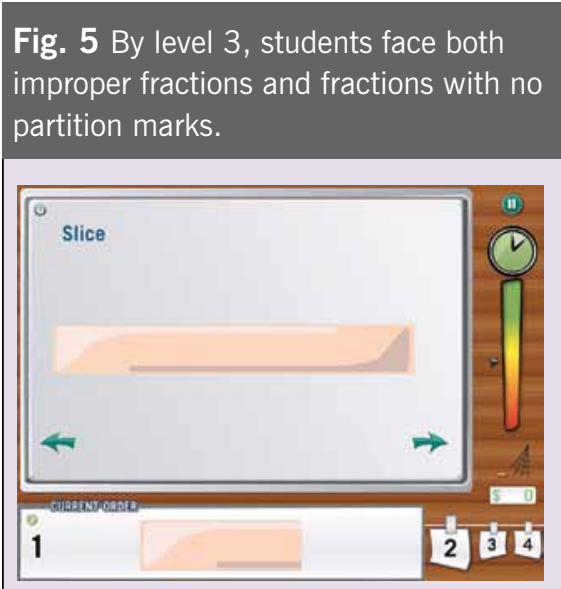

(a)

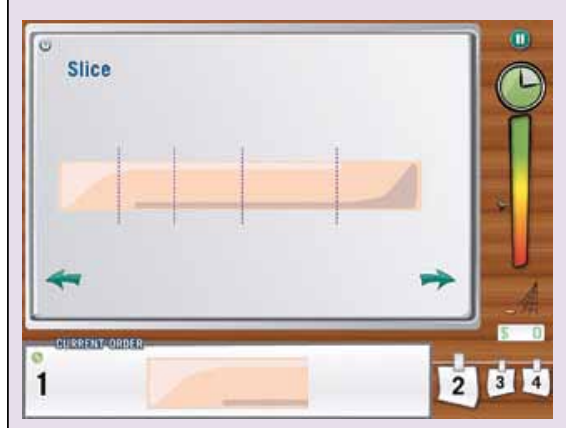

(b)

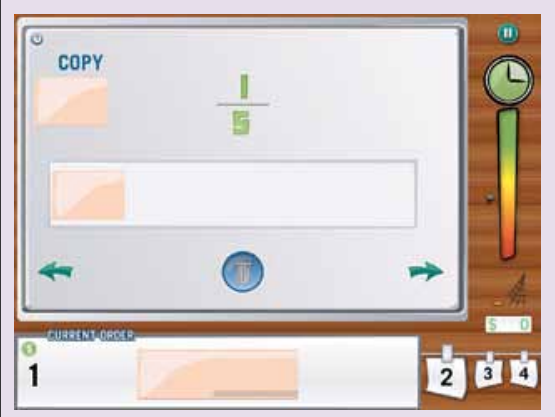

(c)

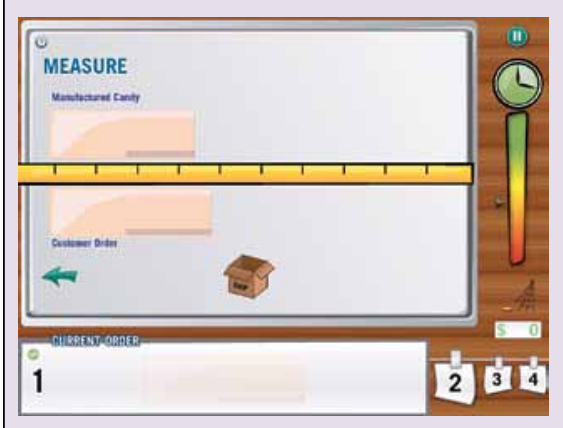

(d) works on the iPhone and iPod touch ${ }^{\circledR}$, as well. For the newer version, look for an icon showing company president, Carmello, as pictured in figure 3 . Here, we describe CandyFactory's first three levels and how they support students' development toward partitive conceptions of fractions.

The goal of the game is to satisfy customer orders for candy bars of specified lengths. Students do this by "slicing" (partitioning) a whole candy bar into some number of pieces and "copying" (iterating) one of those pieces some number of times. At level 1, partitioning marks in the customer order and the whole bar are visible so that students can rely on their part-whole conceptions of fractions to become familiar with the game.

For example, given the situation illustrated in figure $\mathbf{4 a}$, a student can see that the customer order (located at the bottom of the screen) is 6 parts out of 8 equal parts in the whole. The actions of slicing the whole into 8 parts and copying one of those parts 6 times can form a bridge in crossing over from a part-whole view to a partitive view. Note that the student could also satisfy the customer order by producing 4 slices and copying one of them 3 times. In terms of game design, this approach aligns with the basic principle of providing easily achievable challenges early on to instill a sense of success, thus leading to engagement, while introducing game mechanics and the operation of the interface (Schell 2008).

Figure $4 a$ also illustrates several game and interface design decisions made to enhance engagement while scaffolding learning so that the participant can focus on the underlying mathematical principles being conveyed. In terms of game play, the right-hand column contains elements that contribute to the narrative and plot of CandyFactory. From top to bottom, the player sees the shift clock that registers allowable time to complete orders, a customer satisfaction meter that measures speed and accuracy, a bonus meter that figures additional points gained from successfully fulfilling more challenging orders, and a queue of orders at the bottom yet to be filled.

All these features are meant to enhance challenge in the game (Vorderer, Hartmann, and Klimmt 2003). In terms of the interface and interaction, CandyFactory presents a color palette and a look and a feel that the target audience found enjoyable. The interface and interaction also leverage touch, swipe, tap, and other gestural movements that prior work has found to be beneficial for investigating mathematical concepts (Evans et al. 2013)

At level 2, partitioning marks are no longer visible, but the customer orders are always unit fractions of the whole. Students must determine the number of slices needed so that each slice is the same length as the customer order. Students produce slices by swiping their finger from top to bottom across the bar, an action that Apple software developers refer to as a "finger swipe." The main idea for students to learn at this level is the inverse (reciprocal) relationship between the number of slices and the size of each slice. For example,

figure $4 \mathbf{b}$ illustrates a task in which the customer order is $1 / 3$ of the whole. Students can determine this relationship by iterating the length of the customer order within the whole. Then they can produce the customer order from the whole by dividing (with finger swipes) the whole into three parts. Students begin to learn that when the customer order is smaller, it fits into the whole more times and, thus, more slices are needed.

Level 3 resembles level 2, except that it includes nonunit proper 


\section{Early results indicate that the app can effectively increase student engagement and promote an understanding of this partitive view of fractions.}

fractions, meaning that the student must anticipate the coordination of partitioning and iterating (slicing and copying) to produce the customer order. For example, consider the order shown in figure 5a. A student might estimate that the order is two iterations of a $1 / 5$ piece and, thus, slice the whole into five pieces (see fig. $5 \mathbf{b}$ ) and then drag two copies (see fig. 5c) to make $2 / 5$. Although measuring reveals that this is not the correct order (see fig. $\mathbf{5 d}$ ), the student can go back to adjust slicing and copying and, in the process, begin to understand fractions like $2 / 5$ as sizes relative to the whole.

Note that the sequence of actions outlined above fits with research previously described (e.g., Steffe 2001) on how students develop an understanding of the partitive process. Students have to address the size of the given fractional piece relative to the given whole. Whereas at level 2, students can produce the desired piece by imagining how many times it fits into (or iterates within) the whole, at level 3 , students have to anticipate and coordinate partitioning/iterating actions.

On the "Measure" screen, students can reflect on the results of their activity (Simon and Tzur 2004); a ruler is provided to aid a more precise comparison between the produced length and the desired length. This allows students to make adjustments in their coordination of partitioning (slicing) and iterating (copying) actions. We do not expect students (or even experts) to produce the correct fraction on their first try. However, the ways that students adjust their activity are critical to their development of partitive conceptions because they can experience, and begin to anticipate, the size effects of using more or fewer partitions in conjunction with using more or fewer iterations.

\section{CLASSROOM IMPLEMENTATION}

The Learning Transformation

Research Group has begun working with teachers in five middle schools to test the educational value of CandyFactory. Students in those schools played CandyFactory for about twenty minutes, twice per week, for about ten weeks. Pretests and posttests, as well as observations of game play, were used to evaluate gains. Early results indicate that the app can effectively increase student engagement (Evans et al. 2013) and promote an understanding of this partitive view of fractions (Norton, Wilkins, and Boyce 2012).

Teachers in the five schools have used variations of the game to support additional concepts. For example, these teachers have asked students to guess (before slicing or copying) the fractional size of the customer order. Students write down this fraction and then produce it though slicing and copying. Then the students evaluate whether their guess was too large or too small, thus encouraging fraction comparison. If students work with partners, the partner can then try a second guess, going back and forth with a string of ordered fractions until the customer order is satisfied. Teachers can also challenge students to produce the customer order in multiple ways, supporting their conceptions of equivalent fractions.

The Learning Transformation Research Group is continuing to test and improve CandyFactory while designing additional apps to support middle school mathematics. For example, we are in the process of adding a "training mode" to each level, so that students can investigate and reflect on activities at each level without any time pressure, before entering the "evaluation mode" necessary for promotion. We invite readers to visit the project website at http://ltrg.centers.vt.edu/ to get more information about our apps and to send us feedback, which is critical to our efforts to improve the apps.

\section{ACKNOWLEDGMENT}

This work is supported by the $\mathrm{Na}-$ tional Science Foundation (NSF) under Grant No. DRL-1118571, the Institute for Society, Culture and Environment (ISCE) at Virginia Tech, Blacksburg, Virginia, and the Institute for Creativity, Arts, and Technology (ICAT) at Virginia Tech. Any opinions, findings, and conclusions or recommendations expressed in this material are those of the authors and do not necessarily reflect the views of NSF, ISCE, or ICAT. 


\section{REFERENCES}

Common Core State Standards Initiative (CCSSI). 2010. Common Core State Standards for Mathematics. Washington, DC: National Governors Association Center for Best Practices and the Council of Chief State School Officers. http://www.corestandards.org/assets/ CCSSI_Math\%20Standards.pdf

Evans, Michael, Anderson Norton, Mido Chang, Kirby Deckard, and Osman Balci. 2013. "Youth and Video Games: Exploring the Effects of Learning, Achievement, and Engagement.” Zeitschrift für Psychologie 221 (2): 98-106. doi:http://dx.doi .org/10.1027/2151-2604/a000135

Hackenberg, Amy J. 2007. "Units Coordination and the Construction of Improper Fractions: A Revision of the Splitting Hypothesis." Journal of Mathematical Behavior 26 (1): 27-47. doi:http://dx.doi.org/10.1016/j .jmathb.2007.03.002

Howson, Geoffrey. 1995. Mathematics Textbooks: A Comparative Study of Grade 8 Texts. Vancouver: Pacific Education Press.

Norton, Anderson, and Andrea McCloskey. 2008. "Teaching Experiments and Professional Development." Journal of Mathematics Teacher Education 11 (4): 285-305. doi:http://dx.doi .org/10.1007/s10857-008-9076-x

_ 2009. "Modeling Students' Mathematics Using Steffe's Advanced Fractions Schemes." Mathematics Teaching in the Middle School 15 (August): 44-56.

Norton, Anderson, and Jesse Wilkins. 2010. "Mathematical Structures and Mental Structures." In Proceedings of the Thirty-First Annual Meeting of the North American Chapter of the International Group for the Psychology of Mathematics Education: Optimizing Student Understanding in Mathematics, edited by Patricia Brosnan, Diana B. Erchick, and Lucia Flevares, pp. 728-35. Columbus, $\mathrm{OH}$ : Ohio State University.

Norton, Anderson, Jesse L. M. Wilkins, and Steven Boyce. 2012. "Construc- tions of Fractions Schemes: Is There an App for That, Too?" In Proceedings of the Thirty-Third Annual Meeting of the North American Chapter of the International Group for the Psychology of Mathematics Education: Navigating Transitions along Continuums, edited by Laura R. Van Zoest, Jane-Jane Lo, and James L. Kratky, pp. 1135-38. Kalamazoo, MI: University of Western Michigan. Olive, John. 2000. “Computer Tools for Interactive Mathematical Activity in Elementary School.” International Journal of Computers for Mathematical Learning 5 (3): 241-62. doi:http:// dx.doi.org/10.1023/A:1009813723928

Olive, John, and Eugenia Vomvoridi. 2006. "Making Sense of Instruction on Fractions When a Student Lacks Necessary Fractional Schemes: The Case of Tim." Journal of Mathematical Behavior 25 (1): 18-45. doi:http://dx.doi .org/10.1016/j.jmathb.2005.11.003

Schell, Jesse. 2008. The Art of Game Design: A Book of Lenses. Amsterdam: Elsevier/Morgan Kaufmann.

Simon, Martin A., and Ron Tzur. 2004. "Explicating the Role of Mathematical Tasks in Conceptual Learning: An Elaboration of the Hypothetical Learning Trajectory." Mathematical Thinking and Learning 6 (2): 91-104. doi:http://dx.doi.org/10.1207/ s15327833mt10602_2

Steffe, Leslie P. 2001. "A New Hypothesis Concerning Children's Fractional Knowledge." Journal of Mathematical Behavior 20 (3): 267-307. doi:http:// dx.doi.org/10.1016/S0732-3123(02) 00075-5

Steffe, Leslie P., and John Olive, eds. 2010. Children's Fractional Knowledge. New York: Springer. doi:http://dx.doi .org/10.1007/978-1-4419-0591-8

Virginia Tech. 2011. Learning Transformation Research Group (LTRG). "NSF Games Project.” http://ltrg.centers.vt.edu Vorderer, Peter, Tilo Hartmann, and Christoph Klimmt. 2003. "Explaining the Enjoyment of Playing Video Games: The Role of Competition.” In
Proceedings of the Second International Conference on Entertainment Computing ICEC'03, edited by Donald Marinelli, pp. 2-10. Pittsburgh, PA: Carnegie Mellon University.

Watanabe, Tad. 2007. "Initial Treatment of Fractions in Japanese Textbooks.” Focus on Learning Problems in Mathematics 29 (2): 41-60.

Any thoughts on this article? Send an e-mail to mtms@nctm.org._Ed.

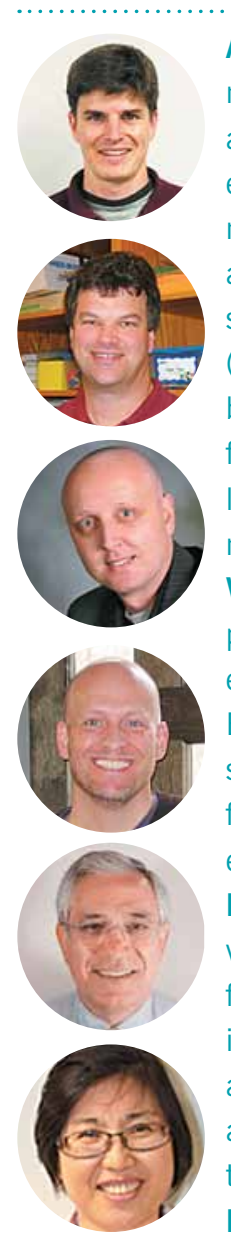

Anderson Norton, norton3@vt.edu, is an associate professor of mathematics education in the mathematics department at Virginia Polytechnic Institute and State University (Virginia Tech) in Blacksburg, Virginia. His research focuses on building psychological models of students' mathematics. Jesse L. M. Wilkins,wilkins@vt.edu, a professor of mathematics education in the School of Education at Virginia Tech, serves as program leader for the $\mathrm{K}$-grade 8 Mathematics Specialist Program. Michael A. Evans, mae@ vt.edu, is an associate professor of learning sciences in the School of Education at Virginia Tech. He serves as principal investigator for the GAMES project. Kirby Deater-Deckard, kirbydd@ vt.edu, a professor of psychology at Virginia Tech, researches children's cognitive development. Osman Balci, balci@vt.edu, is a professor of computer science at Virginia Tech, where he serves as director for the Mobile Software Engineering Lab. Mido Chang, midchang@fiu.edu, is an associate professor at Florida International University in Miami who specializes in the quantitative analysis of educational data. 